\title{
ON SEMICONTINUOUS FUNCTIONS AND BAIRE FUNCTIONS
}

BY

ROBERT E. ZINK(1)

1. According to a classical theorem, every semicontinuous real-valued function of a real variable can be obtained as the limit of a sequence of continuous functions. The corresponding proposition need not hold for the semicontinuous functions defined on an arbitrary topological space; indeed, it is known that the theorem holds in the general case if and only if the topology is perfectly normal [6].

For some purposes, it is important to know when a topological measure space has the property that each semicontinuous real-valued function defined thereon is almost everywhere equal to a function of the first Baire class. In the present article, we are able to resolve this question when the topology is completely regular. A most interesting by-product of our consideration of this problem is the discovery that every Lebesgue measurable function is equivalent to the limit of a sequence of approximately continuous ones. This fact enables us to answer a question, posed in an earlier work [7], concerning the existence of topological measure spaces in which every bounded measurable function is equivalent to a Baire function of the first class.

2. We begin the discussion with some pertinent background material.

The Blumberg upper measurable boundary, $u_{B}$, of a real-valued function $f$ defined on Euclidean $q$-space, $E_{q}$, is specified in the following manner:

If $E_{y}=\{x: f(x)>y\}$, then $u_{B}(\xi)=\inf \left\{y\right.$ : exterior metric density of $E_{y}$ at $\xi$ is zero $\}$.

The lower measurable boundary, $l_{B}$, is similarly defined. Blumberg proved that $u_{B}$ is approximately upper-semicontinuous and that $l_{B}$ is approximately lower-semicontinuous and then observed that approximately semicontinuous functions are Lebesgue measurable. These functions are termed boundaries because each of the sets $\left\{x: u_{B}(x)<f(x)\right\}$ and $\left\{x: l_{B}(x)>f(x)\right\}$ has measure zero, and because the points $\left(\xi, u_{B}(\xi)\right)$ and $\left(\xi, l_{B}(\xi)\right)$ are positively approached by the graph of $f$, for almost every $\xi$. Moreover, if $f$ is measurable, then it coincides almost everywhere with each of its measurable boundaries.

The approximately semicontinuous functions are precisely those functions

Received by the editors September 16, 1963.

(1) While this article was being prepared, the author was supported by grant NSF G-18920 from the National Science Foundation. 
that are semicontinuous with respect to the density topology, $T^{*}$, introduced by Haupt and Pauc [5]. In this topology a set is open if it is measurable and has density one at each of its points; hence, every measurable set contains an equivalent $\boldsymbol{T}^{*}$-open set. We shall make use of the fact, demonstrated in [3], that $T^{*}$ is completely regular.

We shall employ the standard terminology of measure theory. In particular, two measurable sets are said to be equivalent if their symmetric difference has measure zero; two measurable functions are equivalent if they are equal almost everywhere.

The collection of all continuous real-valued functions defined on a topological space $(X, T)$ is denoted by $C(X, T)$.

3. Our principal result concerns the relation between the Lebesgue measurable functions and the Baire functions associated with the density topology. We shall restrict our attention to the consideration of bounded functions, since the case of a general function can be handled easily by first applying the orderpreserving transformation Arctan to the range of the function. It is convenient first to establish two lemmas.

Lemma 1. Let $f$ be a bounded Lebesgue measurable function defined on $E_{q}$, and let $\mathscr{L}$ be the class of all approximately continuous functions that are pointwise bounded above by $f$, i.e.,

$$
\mathscr{L}=\left\{g: g \in C\left(E_{q}, T^{*}\right), g \leqq f\right\} .
$$

If, for each $x$ in $E_{q}$,

$$
s(x)=\sup \{g(x): g \in \mathscr{L}\},
$$

then $f$ is equivalent to the function $s$ thus defined.

Proof. Since $s$ is approximately lower-semicontinuous (that is to say, lowersemicontinuous with respect to $T^{*}$ ), it is measurable, and, by virtue of the definition of $s$, it is certainly clear that $s(x) \leqq f(x)$, for all $x$. Suppose that

$$
m(\{x: f(x)>s(x)\})>0,
$$

where $m$ is the Lebesgue measure. Then there exists a positive number $\varepsilon$ such that

$$
E=\{x: f(x)>s(x)+\varepsilon\}
$$

also has positive measure. From the definition of the density topology follows the existence of a $T^{*}$-open set $U$ contained in and equivalent to $E$. We note that $U$ is nonempty, since it has positive measure.

Now $T^{*}$ is a completely regular topology on $E_{q}$; thus, if $p$ is a point of $U$, then there exists an element $h$ of $C\left(E_{q}, T^{*}\right)$ such that 


$$
h(p)=1, \quad h\left[E_{q}-U\right]=\{0\}
$$

and

$$
0 \leqq h(x) \leqq 1, \quad \text { for all } x \text { in } E_{q}
$$

Let

$$
V=\{x: h(x)>1 / 2\} .
$$

Since $V$ is $T^{*}$-open and nonempty, $m(V)$ is positive. Finally, let

$$
\mathscr{L}_{0}=\{k: k=g+\varepsilon h, g \in \mathscr{L}\},
$$

and, for each $x$ in $E_{q}$, let

$$
s_{0}(x)=\sup \left\{k(x): k \in \mathscr{L}_{0}\right\} .
$$

Clearly, $\mathscr{L}_{0}$ is a subset of $\mathscr{L}$, whence, for all $x$ in $E_{q}$,

$$
s_{0}(x) \leqq s(x) .
$$

But, for each $x$ in $V$, we have

$$
s_{0}(x)>s(x)+\varepsilon / 2 \text {. }
$$

The contradiction thus obtained forces us to reject the assumption that $f(x)$ exceeds $s(x)$ on a set of positive measure, and thus, the first lemma is established.

LeMma 2. For each natural number $n$, there exists an element $g_{n}$ of $\mathscr{L}$ for which

$$
m\left(\left\{x: g_{n}(x)<s(x)-1 / n\right\}\right)<1 / n .
$$

Proof. Suppose that there exists a natural number $n$ such that

$$
m(\{x: g(x)<s(x)-1 / n\}) \geqq 1 / n,
$$

for all $g$ in $\mathscr{L}$. Write

$$
E(g)=\{x: g(x)<s(x)-1 / n\},
$$

and let

$$
a=\inf \{m(E(g)): g \in \mathscr{L}\} .
$$

Let us suppose first that $a<+\infty$. For each positive integer $k$, let $h_{k}$ be an element of $\mathscr{L}$ for which

$$
m\left(E\left(h_{k}\right)\right)<a+1 / k .
$$

We may assume that $\left\{h_{k}\right\}$ is a nondecreasing sequence, for, if the contrary were the case, we should merely replace each $h_{k}$ by $\bigvee_{m=1}^{k} h_{m}$. Then, for all $k$,

and if

$$
E\left(h_{k+1}\right) \subset E\left(h_{k}\right)
$$




$$
E=\bigcap_{k=1}^{\infty} E\left(h_{k}\right) \text {, }
$$

then

$$
m(E)=a
$$

Moreover, for each $g$ in $\mathscr{L}, E-E(g)$ is a null set. Let $U_{0}$ be a $T^{*}$-open subset of $E$ that is equivalent to $E$, let $F$ be the ( $T^{*}$-closed) null set where $u_{B}$, the upper measurable boundary of $f$, differs from $s$ (recall that $s(x)=f(x)$ a.e., by Lemma 1 ) and let

$$
U=U_{0}-F
$$

Then $U$ is $T^{*}$-open, $U$ is contained in $E$ and

$$
m(U)=m(E) .
$$

Let $g$ be any bounded element of $\mathscr{L}$, and let

$$
\alpha=\inf \{s(x)-g(x): x \in U\} .
$$

Choose a point $p$ of $U$ such that

$$
s(p)-g(p)<\alpha+1 / n .
$$

By the complete regularity of $T^{*}$, there exists an element $h$ of $C\left(E_{q}, T^{*}\right)$ satisfying the conditions

$$
h(p)=1, \quad h\left[E_{q}-U\right]=\{0\}
$$

and

$$
0 \leqq h(x) \leqq 1
$$

for all $x$ in $E_{q}$. If

$$
\bar{g}=g+\alpha h,
$$

then $\bar{g}$ is approximately continuous,

$$
\bar{g}\left|E_{q}-U=g\right| E_{q}-U
$$

and, for each $x$ in $U$,

$$
\bar{g}(x) \leqq g(x)+\alpha \leqq s(x)
$$

thus, $\bar{g}$ belongs to $\mathscr{L}$.

Now, $u_{B}-\bar{g}$ is $T^{*}$-upper-semicontinuous so that

$$
V=\left\{x: u_{B}(x)-\bar{g}(x)<1 / n\right\}
$$


is $T^{*}$-open. Moreover, $p$ belongs to $U \cap V$, whence

$$
m(U \cap V)>0 \text {. }
$$

Since, for every $x$ in $U$,

$$
u_{B}(x)=s(x)
$$

it follows that

$$
m(\{x: \bar{g}(x)<s(x)-1 / n\} \cap E)<a .
$$

Since this is inconsistent with the requirement that $E-E(\bar{g})$ be a null set, we have obtained a contradiction.

If $a=+\infty$, then there exists a cube $I$ such that

$$
0<\inf \{m(E(g) \cap I): g \in \mathscr{L}\}<+\infty .
$$

Since the analogue of Lemma 1 holds for the functions defined on $I$, we may derive a contradiction exactly as in the finite case. Thus, we are forced to reject our initial supposition, and, as a consequence, the lemma is proved.

It is now a simple matter to establish our principal theorem.

THEOREM 3. Every bounded Lebesgue measurable function defined on $E_{q}$ is almost everywhere equal to the limit of a nondecreasing sequence of approximately continuous functions.

Proof. For each natural number $n$, let $g_{n}$ be an element of $\mathscr{L}$ such that

$$
m\left(E\left(g_{n}\right)\right)<1 / n,
$$

and let

$$
f_{n}=\bigvee_{k=1}^{n} g_{k}
$$

Let $\varepsilon$ be a specified positive number. For each $n$ exceeding $1 / \varepsilon$,

whence

$$
m\left(\left\{x: f_{n}(x)<s(x)-\varepsilon\right\}\right) \leqq m\left(E\left(g_{n}\right)\right)<1 / n,
$$

$$
m\left(\left\{x: \lim _{n} f_{n}(x)<s(x)-\varepsilon\right\}\right)=0
$$

Since $\varepsilon$ may be taken to be arbitrarily small, it follows that

$$
\lim _{n} f_{n}(x) \geqq s(x), \text { a.e. }
$$

Finally, the equivalence of $s$ and $f$ implies the equivalence of $f$ and $\lim _{n} f_{n}$.

COROllaRy 4 (VITAli-CARATHÉODORY). Every bounded Lebesgue measurable function is almost everywhere equal to an element of the second Baire class. 
Proof. It is necessary only to recall that the approximately continuous functions are all of Baire type 1 [2]. The desired result is then an elementary consequence of the theorem.

4. We turn now to the consideration of an application of the foregoing ideas to the classification of measure spaces problem.

A topological measure space $(X, \boldsymbol{T}, \mathbf{\Sigma}, \mu)$ is a topological space $(X, \boldsymbol{T})$ and a measure space $(X, \Sigma, \mu)$ such that $T$ is a subset of $\boldsymbol{\Sigma}$ and $\mu(U)$ is positive for each nonempty element $U$ of $T$. In [7] we have classified measure spaces of this type by first singling out certain classes of topologically related functions and then observing whether the bounded measurable functions are equivalent to members of one of these classes. For example, a topological measure space is termed a 0 -space if each bounded measurable function is almost everywhere equal to a continuous function; it is a 1 -space if it is not a 0 -space and if each bounded measurable function is equivalent to a lower-(upper-)semicontinuous function; it is a 2 -space if it is neither a 1-space nor a 0 -space and if every bounded measurable function is equivalent to the limit of a sequence of lower-(upper-) semicontinuous ones. The spaces of type $\alpha$ for countable ordinals $\alpha$ are defined inductively. This system of classification proved to be a most useful one, since in the case of finite ordinals, we found that $\alpha$-spaces can be characterized by certain smoothness properties of the measures associated with them. In this connection, the following theorem is typical.

THEOREM 5. Let $(X, T, \Sigma \mu)$ be a topological measure space. In order that

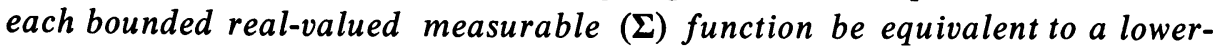
semicontinuous one, it is both necessary and sufficient that each measurable set be equivalent to an open set.

Another natural classification scheme follows the same general pattern but uses as approximating functions the elements of the various Baire classes; thus, a topological measure space is called a $B_{\alpha}$-space if each of the bounded measurable functions associated therewith is equivalent to a function of Baire type $\alpha$ and if $\alpha$ is the smallest ordinal for which this is true. In the general case, this system seems to be more difficult to work with than is the one described earlier; however, in the presence of a suitably strong topological separation axiom, the technique developed for the demonstration of Theorem 3 can be used to show that the two systems are identical.

LEMMA 6. Let there be imposed on the topological measure space $(X, T, \Sigma, \mu)$ the additional condition that $\boldsymbol{T}$ be completely regular. Then, every bounded semicontinuous function on $X$ is almost everywhere equal to a function belonging to the first Baire class, and every bounded element of the first Baire class is equivalent to a lower-(upper-)semicontinuous function. 
Proof. If $u$ is bounded and upper-semicontinuous, then the arguments given in establishing the lemmas that precede Theorem 3 can be duplicated here to show that $u$ is equivalent to the limit of a nondecreasing sequence of continuous functions. By making use of the fact that the negative of a lower-semicontinuous function is upper-semicontinuous, it is easy to see that a bounded lower-semicontinuous function is almost everywhere equal to the limit of a nonincreasing sequence of continuous functions.

Now let $f$ be a bounded function of Baire type 1. Suppose that $f=\lim _{n} f_{n}$, where each $f_{n}$ is continuous. Without loss of generality, we may assume that the $f_{n}$ are uniformly bounded (by the bounds of $f$, for example), in which case each of the functions

$$
g_{n}=\bigvee_{k=n}^{\infty} f_{k}
$$

is bounded also. Since each $f_{n}$ is, in particular, lower-semicontinuous, it follows that the $g_{n}$ are also lower-semicontinuous. According to the proposition established in the preceding paragraph, there exists, for each natural number $n$, a nonincreasing sequence $\left\{g_{n k}\right\}_{k=1}^{\infty}$ of continuous functions converging almost everywhere to $g_{n}$. Since

$$
\begin{aligned}
f & =\lim _{n} \sup f_{n} \\
& =\bigwedge_{n=1}^{\infty} \bigvee_{k=n}^{\infty} f_{k} \\
& =\bigwedge_{n=1}^{\infty} g_{n},
\end{aligned}
$$

it is clear that $f$ is equivalent to the upper-semicontinuous function

$$
g=\bigwedge_{n=1}^{\infty} \bigwedge_{k=1}^{\infty} g_{n k} .
$$

THEOREM 7. A completely regular topological measure space is a $B_{1}$-space if and only if it is a 1-space.

Proof. The theorem is an immediate consequence of the definitions and Lemma 6.

In [7] we gave an example of a denumerable $B_{1}$-space and raised the question of the existence of a nonatomic space of this type. Theorem 7 now enables us to answer that question affirmatively.

EXAMPLE 8. Let $(I, L, m)$ be the Lebesgue measure space associated with the unit interval, and let $T^{*}$ be the density topology on $I$. By virtue of Theorem 5 , $\left(I, T^{*}, L, m\right)$ is a 1 -space. Since $T^{*}$ is completely regular, a direct application 
of Theorem 7 shows that $\left(I, T^{*}, L, m\right)$ is also an example of an (atom-free) $B_{1}$-space.

Within the general framework of a completely regular topological measure space, Lemma 6 constitutes the first step in an inductive proof of the measuretheoretic equivalence of the finite-ordinal Baire classes and the corresponding classes of functions generated by the semicontinuous functions. In order to simplify the discussion, we introduce a system of notation for the latter classes. Let the class of lower-semicontinuous functions be denoted by $\mathscr{L}_{1}$. If $\alpha$ is an ordinal number that exceeds 1 and if the function classes $\mathscr{L}_{\beta}$ have been defined for each $\beta$ less than $\alpha$, let $\mathscr{L}_{\alpha}$ denote the class of all limits of convergent sequences of functions belonging to $\bigcup_{\beta<\alpha} \mathscr{L}_{\beta}$. In a similar fashion, the classes generated by the upper-semicontinuous functions are denoted by $\mathscr{U}_{1}, \mathscr{U}_{2}, \cdots$.

THEOREM 9. Let $(X, T, \Sigma, \mu)$ be a completely regular topological measure space. For each finite positive ordinal number $\alpha$, every bounded function of Baire type $\alpha$ is equivalent to an element of $\mathscr{L}_{\alpha}\left(\mathscr{U}_{\alpha}\right)$, and every bounded function belonging to $\mathscr{L}_{\alpha}\left(\mathscr{U}_{\alpha}\right)$ is equivalent to a Baire function of the $\alpha$ th class.

Proof. We shall consider only the classes $\mathscr{L}_{\alpha}$. The proof employs the method of finite induction. In view of Lemma 6, we need provide only the induction step. Suppose then that the proposition has been established for a given finite positive ordinal $\alpha$. Let $f$ be a bounded Baire function of type $\alpha+1$, and let $\left\{f_{n}\right\}$ be a sequence of bounded functions of Baire type $\alpha$ that converges to $f$. By virtue of the induction hypothesis, there exists, for each natural number $n$, an element $g_{n}$ of $\mathscr{L}_{\alpha}$ that is equivalent to $f_{n}$. Now if $\alpha$ is odd, then the supremum of a sequence of elements of $\mathscr{L}_{\alpha}$ is again an element of $\mathscr{L}_{\alpha}$, while if $\alpha$ is even, then the infimum of such a sequence belongs once more to $\mathscr{L}_{\alpha}$. Hence, at least one of the functions limsup $g_{n}$ and $\liminf _{n} g_{n}$ belongs to $\mathscr{L}_{\alpha+1}$. Since $f$ is equivalent to both of these functions, the first half of the desired result has been obtained.

Now let $f$ be a bounded member of $\mathscr{L}_{\alpha+1}$. If $\alpha$ is even, then there exists an increasing sequence $\left\{f_{n}\right\}$ of bounded elements of $\mathscr{L}_{\alpha}$ having limit $f$. According to the induction hypothesis, each $f_{n}$ is equivalent to a function $g_{n}$ of Baire type $\alpha$. In order to save ourselves from future embarrassment, we suppose, without loss of generality, that the $g_{n}$ are uniformly bounded by the bounds of $f$. For each natural number $n$, let

$$
h_{n}=\bigvee_{k=1}^{n} g_{k} .
$$

Then $\left\{h_{n}\right\}$ is a convergent sequence of elements of the $\alpha$ th Baire class, and, for each $n, h_{n}$ and $f_{n}$ are equivalent. Thus, $f$ is equivalent to $\lim _{n} h_{n}$, a function of Baire type $\alpha+1$. A similar argument is sufficient to dispatch the case in which $\alpha$ is odd. 
We conclude the article with the obvious application of Theorem 9 to the classification problem we have been considering.

COROLlaRy 10. Let $\alpha$ be a finite ordinal number. In order that a completely regular topological measure space be an $\alpha$-space, it is both necessary and sufficient that it be a $B_{\alpha}$-space.

\section{REFERENCES}

1. H. Blumberg, The measurable boundaries of an arbitrary function, Acta Math. 65 (1935), 263-282.

2. A. Denjoy, Sur les fonctions dérivées sommable, Bull. Soc. Math. France 43 (1916), 161-248.

3. C. Goffman, C. J. Neugebauer and T. Nishiura, Density topology and approximate continuity, Duke Math. J. 28 (1961), 497-506.

4. C. Goffman and D. Waterman, Approximately continuous transformations, Proc. Amer. Math. Soc. 67 (1961), 116-121.

5. O. Haupt and C. Pauc, La topologie approximative de Denjoy envisagée comme vraie topologie, C. R. Acad. Sci. Paris 234 (1952), 390-392.

6. K. Nagami, Baire sets, Borel sets and some typical semi-continuous functions, Nagoya Math. J. 7 (1954), 85-93.

7. R. E. Zink, A classification of measure spaces, Colloq. Math. (to appear).

Purdue University,

LAFAYETTE, INDIANA 